found that delayed recovery at six weeks was associated with psychological vulnerability in patients who had had influenza, White et al (in press) found that only patients with glandular fever, but not other viral infections were associated with fatigue at six months follow up. Wessely et al (1995) in a large prospective study, found no evidence that common infections were related to the development of CFS in primary care.

Second, Shanks \& Ho-Yen claimed that counselling resulted in an $80 \%$ improvement rate. While this result is very impressive the authors failed to provide any data which supports their sweeping conclusion. As one of the defining characteristics of CFS (Sharpe et al, 1991) is a $50 \%$ reduction in activity levels it is difficult to see how $80 \%$ of the group studied could have improved in terms of their disability when they were given advice to live within their limits.

Imbodin, J., Canter, A. \& Ciuff, L. (1961) Convalescence from influenza: a study of the psychological and clinical determinants. Archives of Internal Medicine, 108, 115-121.

Shanks, M. F. \& Ho-YeN, D. O. (1995) A clinical study of chronic fatigue syndrome. British Journal of Psychiarry, 166, 798-801.

Sharpe, M., Archard, L.. Banatvala, J., et al (1991) Chronic fatigue syndrome: Guidelines for research. Journal of the Royal Society of Medicine. 84, 118-121.

Wesseley, S., Chalder, T.. Pawlikowska. T., et al (1995) Postinfectious fatigue: prospective study in primary care. Lancet. 345, 1333-1338

Whitr, P. D., Thomas, J. M., Amess, J. S., et al (1995) The existence of a fatigue syndrome after glandular fever. Psychological Medicine, in press.

King's College School of Medicine

T. Chalder

\& Institute of Psychiatry

London SE5 $8 A Z$

AUTHOR'S REPLY: Ms Chalder is correct in saying that our findings do not prove that the evidence of viral infection detected in over half our sample of patients was closely connected with the subsequent chronic fatigue syndrome. In fact we showed that CFS patients with and without associated emotional disorder were biologically similar over a range of viral and immunological measures. This confirmed previous studies which showed that such measures, while they might contribute operationally to clinical definition, have little diagnostic value in this population far less any established etiological significance. Our reference to 'precipitating' viral infection was meant in the sense of association with the onset of symptoms, rather than implying aetiology, which of course could be in either direction.

Counselling to remain within energy limits is not logically incompatible with progressive diminution of the symptoms of fatigue. The detailed findings related to psychiatric diagnosis were not included in our paper, but in this early onset population generalised anxiety and panic disorders were much more frequently identified than the chronic depressive states described in populations with a longer duration of fatigue, and constituted over half the case diagnoses.

Patients with more recent onset of symptoms and a different pattern of associated emotional disorder appear to have a better prognosis. Admittedly this finding is based on a global clinical severity scale and simple data about occupation, although at the time the study was planned, more sophisticated assessments of fatigue symptomatology had not been developed.

Royal Cornhill Hospital

M.F. SHanks Aberdeen $A B 92 Z H$

\section{Blu-Tack - a novel substance of abuse}

SIR: We wish to report a novel form of substance abuse, namely the smoking of the adhesive material Blu-Tack. Three male patients (aged 23, 27 and 32 years) detained on our semi-secure unit for the chronic mentally ill, all with the diagnoses of paranoid schizophrenia and substance abuse, were found to have been attempting this activity in the enforced absence of their preferred substances.

Two methods were employed. Both commenced with drying the Blu-Tack on a radiator for three weeks (or until the experimenter's patience expired). The resulting residue was then either crumbled and smoked with tobacco in a roll-up cigarette, or inhaled from the burning end of a match. One of the three informed us that he experienced a very slight "high" along with a peculiar sensation in his stomach (the latter may however be related to his longstanding somatic delusions), but denied any other effects. Another (the originator, claiming to have thought of the procedure himself) found it to have virtually no effect on him. The third also found it an unrewarding activity. No changes were observed in these patients' mental states or physical conditions as a result of this particular activity.

Neither the hospital pharmacy nor the Regional Poisons Centre had any information regarding this phenomenon, and there does not appear to be any reference to it in the medical literature. The only other report we have heard emanates from HM Prison Saughton (CPN Ian Wishart, personal communication). 
When contacted, the manufacturers (Bostik) reported that Blu-Tack's constituents could give rise to glaucoma, lung damage and ultimately death if abused. In order to determine the constituents responsible for any psychotropic effect and clarify the nature of any potential toxicity, we requested an analysis of Blu-Tack residue from the Department of Chemistry at the University of Edinburgh. Electron ionisation mass spectrometry was unhelpful, but useful results were obtained from pyrolysis of a dried sample followed by gas chromatography separation and further mass spectrometry. A complex mixture of hydrocarbons was revealed, whose principal constituents included butene, heptadiene, propene, pentene and hexadiene. It may therefore be presumed that Blu-Tack smoking has attractions and risks similar to those of inhaling lighter gas, a common and well-documented phenomenon.

Blu-Tack is fairly readily available via art groups, noticeboards and wall posters even in a locked ward setting. In view of the above findings and the consequent possibility of serious risk to the physical and mental health of those attempting this activity, we wish to draw attention to it as a novel substance of abuse.

We wish to thank Professor John Monaghan (Department of Chemistry, The University of Edinburgh) for his helpful advice. and $\mathrm{Mr}$ W. E. Morden (I.C.I.C. \& P. Ltd, Runcorn Heath, Cheshire) for performing the analysis.

\section{WALKER-KINNEAR}

T. JONES

Royal Edinburgh Hospital

Edinburgh EHIO SHF

\section{Scale for assessing hedonic tone}

SIR: Snaith et al (1995) have developed a new pleasure scale (the Snaith Hamilton Pleasure Scale, SHAPS) corresponding to the need for a simple scale, not affected by socio-demographical factors and allowing an easy translation in other languages. They criticised the existing pleasure scales (Fawcett Clark Pleasure Capacity Scale or FCPCS; Chapman's scales) that are too long and presented cultural bias. Indeed several studies (Loas et al, 1992) have shown a weak discriminant validity of the FCPCS in other cultures mainly due to differences in cultural backgrounds and in socioeconomic levels. Consequently we have built up (Loas et al, 1994) a shortened version of the FCPCS containing 12 items assessing only sensorial and physical features of pleasure. These items can be considered as being less sensitive to cultural biases.

In two studies including both healthy subjects $(n=314)$ and patients with major depressive dis- order $(n=103)$ we demonstrated that the subscale (FCPCS-PP) has good construct and discriminant validities and a satisfactory reliability (Loas $e t$ al, 1994, 1995).

Loas, G., Salinas, E., Guelfi, J. D., et al (1992) Physical anhedonia in major depressive disorder. Journal of Affective Disorders, 25, 139-146.

_, - Pierson, A., et al (1994) Anhedonia and blunted affect in major depressive disorder. Comprehensive Psychiatry, 35, 366-372.

-, Boyer, P., Fremaux, D., et al (1995) The physical pleasuredispleasure scale (P-PDS): Study of validation on 295 subjects. European Review of Applied Psychology, in press.

Snaith, R. P., Hamilton, M., Morley, S., et al (1995) A scale for the assessment of hedonic tone. The Snaith-Hamilton Pleasure Scale. British Journal of Psychiatry, 167, 99-103.

Service Hospitalo-Universitaire de Psychiatrie

Hopital Pinel

80044 Amiens cedex 01

France

INSERM, 100 rue de la Santé

P. BOYER

75674 Paris cedex 14

France

\section{Neuroleptic associated extrapyramidal symptoms}

SIR: It is generally agreed that, according to the dopamine hypothesis, the antipsychotic effect of neuroleptics is mediated by blockade of mesolimbic or mesocortical $\mathrm{D}_{2}$ dopamine receptors whereas the mechanism by which neuroleptic drugs cause extrapyramidal side-effects is not yet entirely understood. It has recently been established that Parkinson's disease, which is clinically similar to neuroleptic-induced parkinsonism, is associated with oxidative damage due to mitochondrial dysfunction (Schapira, 1994). A defect of complex I of the mitochondrial respiratory chain has been reported in platelets, muscle and brain tissue of patients with Parkinson's disease. MPTP (1-methyl4-phenyl-1,2,3,6-tetrahydropyridine) which may cause Parkinson's disease, inhibits complex I of the respiratory chain. It is a substrate for the dopamine reuptake pathway and is concentrated into mitochondria. It seemed therefore possible that neuroleptics might cause extrapyramidal side effects via a similar mechanism to that of MPTP, especially since haloperidol is structurally similar to MPTP and may be converted to a haloperidol pyridinium product analogous to $\mathrm{MPP}^{+}$. Further evidence was derived from studies in rats which showed that neuroleptics block complex I of the respiratory chain in vitro (Burkhardt et al, 1993). We therefore 